\title{
Design of practical sliding-mode controllers with constant switching frequency for power converters
}

\author{
Eva M. Navarro-López ${ }^{\mathrm{a}, *}$, Domingo Cortés $^{\mathrm{b}}$, Christian Castro $^{\mathrm{c}}$ \\ a School of Computer Science, Centre for Interdisciplinary Computational and Dynamical Analysis, The University of Manchester, \\ Oxford Road, Kilburn Building, Manchester M13 9PL, UK \\ b Sección de Mecatrónica, Departamento de Ingeniería Eléctrica, CINVESTAV-IPN, Av. IPN 2508, Col. San Pedro Zacatenco, 07360 Mexico City, Mexico \\ ${ }^{\mathrm{c}}$ Centro de Investigación en Computación del IPN, Av. José Othón de Mendizábal s/n, Col. Nueva Industrial Vallejo, 07738 Mexico City, Mexico
}

\section{A R T I C L E I N F O}

\section{Article history:}

Received 4 December 2007

Received in revised form 30 July 2008

Accepted 26 October 2008

Available online 13 December 2008

\section{PACS:}

84.30.Jc

02.30.Yy

07.05.Dz

07.50.Ek

47.27.ed

02.30.Hq

01.50.My

Keywords:

Power converters

Sliding-mode control

Pulse-width modulation

Nonlinear systems

Discontinuous control

\begin{abstract}
A B S T R A C T
A novel experimentally motivated method in order to design a family of easy-to-implement sliding-mode controllers for power converters is proposed. Two main results are presented. First, the relation between sliding-mode control and average control is reinterpreted so that the limitation of the switching frequency for the closed-loop system is achieved in a more direct way than other methods so far reported in the literature. For this purpose, a class of sliding surfaces which makes the associated equivalent control be the system average control is proposed. Second, the achievement of a constant switching frequency in the controlled system is assured without requiring the sliding-mode-based controller to be modified, unlike most previous works. As a result, the proposed sliding surfaces-type can be directly implemented via a pulse-width modulator. The control methodology is implemented for the voltage control in a boost converter prototype in which the load is considered unknown. Experimental results confirm high performance and robustness under parameters variation. Furthermore, the solution proposed is easy to implement and well-suited for other power converters.
\end{abstract}

(c) 2008 Elsevier B.V. All rights reserved.

\section{Introduction}

The main goal of this paper is to design a class of easy-toimplement sliding-mode controllers for power converters in which the control switches with a fixed known frequency established by the designer. For this purpose, a new type of switching surfaces is proposed. These surfaces make the equivalent control be the average control and consequently, they can be directly implemented by means of a pulse-width modulator (PWM).

Sliding-mode control (SMC) has been proven to be effective in a wide range of practical control problems with nonlinear complex dynamics [1]. Such a success is mainly due to the relatively simple design procedure, the good con-

\footnotetext{
* Corresponding author. Tel.: +44161275 6209; fax: +44 1612756204 . E-mail addresses: eva.navarrolopez@gmail.com

(E.M. Navarro-López),domingo.cortes@gmail.com, dcortes@cinvestav.mx

(D. Cortés), naruto.christian@gmail.com (C. Castro).
}

trolled system performance, in addition to the robustness under input perturbations and variations of system dynamic properties.

The main characteristic of systems exhibiting sliding modes is their discontinuous nature. The sliding motion can be either intrinsic to the system or be induced in it by means of a discontinuous control. SMC is particularly appropriate for power electronics systems because these are intrinsically discontinuous. This fact has attracted the interest of many power electronics engineers, who have applied SMC to power converters.

The SMC-design process gives an expression for the switch position in power converters. In the ideal case, the switching frequency is infinity, which obviously cannot be achieved. Nevertheless, the higher the switching frequency is, the better the approximation to an ideal sliding mode is. However, due to the characteristics of the actual switch, an upper limit for this switching frequency must be imposed. In many applications, having a constant switching frequency is desirable, but sometimes is mandatory. Due to this fact, methods to limit the switching frequency have been proposed. In 
Section 2, where the SMC-design process is revisited, these methods are discussed.

The implementation of SMC for power converters usually involves two steps. First, to design an ideal SMC by means of an ideal sliding surface. Second, to modify the sliding surface in order to implement the control with a constant switching frequency, or at least with an upper limit for the switching frequency. Ideal SMC algorithms are usually simple, however, its implementation becomes difficult due to the necessity of the second design step. This drawback is overcome in this paper. If a controller has the form specified in this paper then it can be straightforwardly implemented assuring a constant switching frequency, without requiring the control law to be modified.

The problem of achieving a constant switching frequency has been addressed in several works [2-11]. Most of these solutions are based on a relation between the SMC and the average control. Such a relation arises from the physical meaning of the equivalent control associated with a SMC. A different interpretation of the SMC-average-control relation is given in Section 2, which will be the basis for proposing a new class of controllers in Section 3.

The SMC proposed in this paper is based on a class of sliding surfaces which can be directly implemented by using a PWM, and, hence, with a constant switching frequency. The main idea followed is that one surface in which the system has desired dynamics is akin to be embedded into a sliding surface. In [12], following the work in [13], a preliminary sketch of this proposal was presented. Nevertheless, in the present paper, the validity of the sliding-modebased control methodology is analytically proven.

The idea of using a surface in order to build the sliding surface for the closed-loop system has been successfully implemented in a boost-converter prototype. The simulations and experimental results for the example show that the controller is easy to implement and yet is robust under load and input voltage variations.

\section{Relation between sliding-mode controllers and average controllers in power electronics}

Power converters can be modelled by

$\dot{\mathbf{x}}=f(\mathbf{x})+g(\mathbf{x}) u$,

where $\mathbf{x} \in \mathbb{R}^{n}$ is the system state vector, usually consisting of inductor currents and capacitor voltages. $f$ and $g$ are continuous vector fields and $u \in\{0,1\}$ is the switch position which makes the system discontinuous. The dot stands for the derivative with respect to time.

The SMC-design process aims at determining the switch position $u$, which generally has the form,

$u= \begin{cases}0 & \text { if } \sigma(\mathbf{x}, t)<0 \\ 1 & \text { if } \sigma(\mathbf{x}, t)>0\end{cases}$

where $\sigma$ is a smooth scalar function. Since sliding-mode controllers are intrinsically discontinuous, it is natural to use a discontinuous system model for designing these controllers.

If a sliding-mode controller works then, at some time, the system trajectory will evolve on the surface $\sigma=0$. When this happens, the system trajectory is described by means of the equivalent dynamics [14,15],

$\dot{\mathbf{x}}=f(\mathbf{x})+g(\mathbf{x}) u_{\mathrm{eq}}$,

where $u_{\text {eq }}$, referred to as the equivalent control, is the solution for $u$ of the equation $\dot{\sigma}=0$. Dynamics described by (3) are valid just in the ideal case of infinity switching frequency. In a more practical case of having a high but limited switching frequency, the system trajectory does not evolve on the surface $\sigma=0$ but in a boundary layer [15]. In this case, (3) can be written as

$\dot{\tilde{\mathbf{x}}}=\mathbf{f}(\tilde{\mathbf{x}})+\mathbf{g}(\tilde{\mathbf{x}}) \mathbf{u}_{\mathbf{e q}}$,

where $\tilde{\mathbf{x}}$ is the average of $\mathbf{x}$.

Controller (2) cannot be directly implemented because, in this case, the frequency would operate in free run, just limited by physical constraints of the switch element. To have a good efficiency and to protect the switch element, among other practical considerations, the switching frequency must be assured to have an upper limit. To this end, a hysteresis band could be used in (2) instead of the sign of $\sigma$. Alternatively, the switch could be assured to be in on (off)-mode for a constant time and modulate the on (off)-time. In some applications, it is highly convenient, and even mandatory, to have a constant switching frequency. The hysteresis band and constant on-time can only lead to a constant switching frequency in the stationary state.

On the other hand, average control is based on the model

$\dot{\tilde{\mathbf{x}}}=\mathbf{f}(\tilde{\mathbf{x}})+\mathbf{g}(\tilde{\mathbf{x}}) \mathbf{d}$.

Vector $\tilde{\mathbf{x}}$ consists of the average of inductor currents and capacitors voltages; $f$ and $g$ are the same continuous vector fields than those appearing in (1); $d \in[0,1]$ is the duty cycle.

The average-controller design process yields to an expression for the duty cycle,

$d=\frac{\operatorname{Num}(\tilde{\mathbf{x}})}{\operatorname{Den}(\tilde{\mathbf{x}})}$.

This signal must be passed through a PWM to carry out the actual switching.

In [15], it is proven that $u_{\text {eq }}$ is the average of $u$. Hence, the performance of a sliding-mode controller $u$ should be like the performance of an average controller $d$ provided that $u_{\mathrm{eq}}=d$. This idea is proven in [16]. Consequently, it is posible to design a sliding-mode controller as that defined in Eq. (2) and implement it with a PWM by

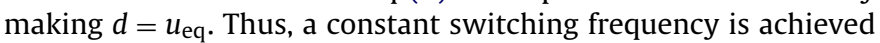
(Fig. 1). This idea is the basis of several works [3,4,7,9,11]. The problem is that the implementation of $u_{\mathrm{eq}}$ is usually far more difficult than $\sigma$. By using the equivalence between $u_{\text {eq }}$ and $d$, an alternative method to simplify the sliding-mode controller implementation is proposed in [9]. Instead of using a PWM in order to achieve (6), it could be assured that $\operatorname{Den}(\tilde{\mathbf{x}}) u_{\text {eq }}=\operatorname{Num}(\tilde{\mathbf{x}})$, and therefore, there is no need to use a divisor. However, for this purpose, it is necessary that the PWM has a sawtooth with variable amplitude. All

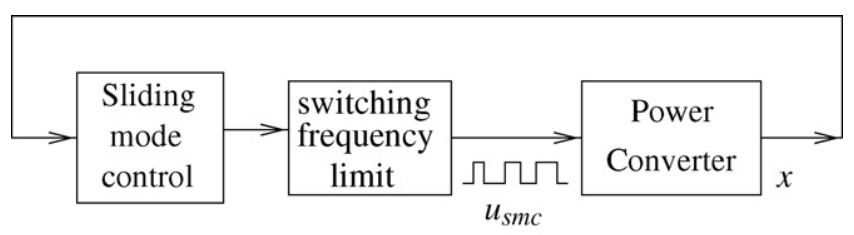

(a) Sliding mode control implementation

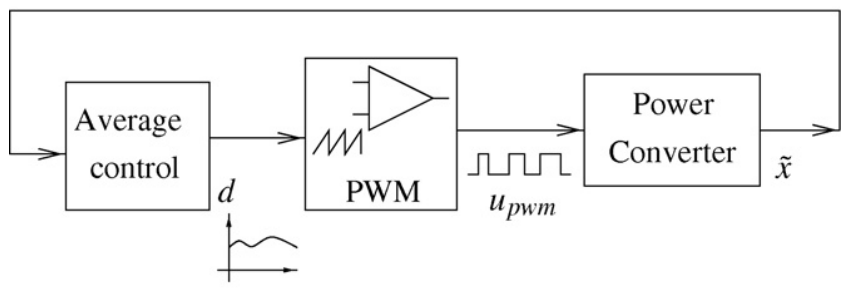

(b) Average control implementation

Fig. 1. Relation between SMC and average controls: if $d=u_{\mathrm{eq}}$ then $u_{\mathrm{smc}}$ and $u_{\mathrm{pwm}}$ are similar. 


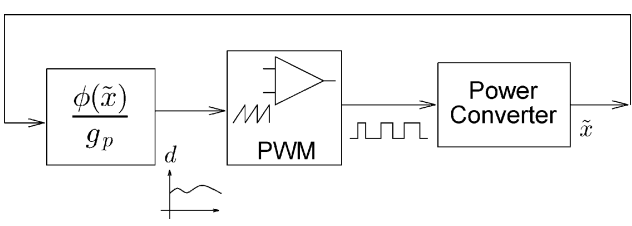

Fig. 2. Implementation of function $\sigma$ (9), defining the surface $\sigma=0$, by means of $\phi$.

these works are based on proposing the sliding surface $\sigma=0$ and on finding a way to implement it.

This paper is also based on this idea, however, our main goal is obtaining easy-to-implement sliding surfaces. For this purpose, in the next section, a new class of sliding surfaces $\sigma=0$ is proposed.

\section{Experimentally motivated sliding-mode-based control methodology}

This section aims at answering the following question: what is (if any) the sliding-mode controller that implements the average controller (6)? That is, what is the expression for $\sigma$ such that the equivalent control associated with the sliding surface $\sigma=0$ is $u_{\mathrm{eq}}=$ d?

According to (6), $\operatorname{Num}(\tilde{\mathbf{x}})-\operatorname{Den}(\tilde{\mathbf{x}}) d=0$, that is, $\operatorname{Num}(\tilde{\mathbf{x}})-$ $\operatorname{Den}(\tilde{\mathbf{x}}) u_{\mathrm{eq}}=0$. Since $u_{\mathrm{eq}}$ is the solution for $u$ of $\dot{\sigma}(\mathbf{x}, t)=0$, it can be said that,

$\dot{\sigma}=k_{a}(\operatorname{Num}(\mathbf{x})-\operatorname{Den}(\mathbf{x}) u(\mathbf{x}))$,

and consequently,

$\sigma=k_{a} \int_{0}^{t}(\operatorname{Num}(\mathbf{x}(\tau))-\operatorname{Den}(\mathbf{x}(\tau)) u(\tau)) d \tau+k_{b}$,

where $k_{a}$ and $k_{b}$ are constants. Note that $u_{\text {eq }}$ is the same, independently of $k_{a}$ and $k_{b}$ values.

To conclude with, if an average controller $d$ given by (6) corresponds to an equivalent control associated with a sliding-mode controller (2) then the associated sliding surface is given by $\sigma=0$, with $\sigma$ as defined in (8).

The following considerations are made

$\operatorname{Num}(\mathbf{x})=\phi(\mathbf{x})$ and $\operatorname{Den}(\mathbf{x})=g_{p}$,

with $\phi$ a smooth scalar function and $g_{p}>0$ the PWM gain. Hence, $\sigma$ in (8) can be rewritten as

$\sigma=k_{a} \int_{0}^{t}\left(\phi(\mathbf{x}(\tau))-g_{p} u(\tau)\right) d \tau+k_{b}$

This is an interesting case, because a sliding surface $\sigma=0$ with $\sigma$ of the form (9) can be easily implemented with a PWM just making $d=\phi(\mathbf{x}) / g_{p}=u_{\text {eq }}$ (Fig. 2). Therefore, sliding-mode controllers of the form (2) and (9) are easy to implement with constant switching frequency.

It is important to understand that what has been proposed is a structure for sliding surfaces that can be easily implemented with a constant switching frequency via a PWM. For each application case, the designer must propose $\phi(\mathbf{x})$ and make sure that $\sigma=0$ with $\sigma$ as in (9) is indeed a sliding surface, that is, that all the conditions for a sliding mode to exist are accomplished. The analytical proof for this assessment must be done for each function $\phi(\mathbf{x})$ defined, and consequently, for each type of converter treated.

Thus, the sliding-mode-based control methodology proposed consists of three steps:

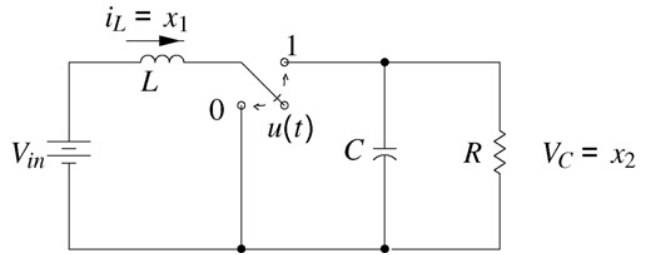

Fig. 3. Simplified diagram of the boost converter.

Step 1. A surface along which the system would have desired dynamics is proposed. This surface is $\phi(\mathbf{x}, t)=0$.

Step 2. A sliding surface is proposed. This surface can be implemented directly by inserting $\phi(\mathbf{x}, t) / g_{p}$ into a PWM. This surface is $\sigma(\mathbf{x}, t)=0$ with $\sigma$ as proposed in (9). The surface $\phi=0$ is akin to be embedded into the sliding surface $\sigma=0$.

Step 3. The converter and controller parameters are designed so that the system trajectories enter a sliding regime on $\sigma(\mathbf{x}, t)=0$ by using control (2). In addition, the trajectories are assured to evolve towards the desired equilibrium on this surface.

In the next section, this sliding-motion-based control scheme is applied to the voltage control of a boost converter.

\section{Example: Voltage control for the boost converter}

\subsection{Proposal of the switching strategy for the boost converter}

A boost converter, which simplified diagram is shown in Fig. 3, is considered. The circuit objective is to keep a desired constant output-voltage $\left(V_{\text {ref }}\right)$ regardless of the input voltage $\left(V_{\text {in }}\right)$ and load variations. From the diagram, a linear model can be obtained for each switch position. Combining both models into a single one, the following system is obtained,

$\dot{x}_{1}=\frac{V_{\text {in }}}{L}-u \frac{x_{2}}{L}$,

$\dot{x}_{2}=-\frac{x_{2}}{R C}+u \frac{x_{1}}{C}$

where $x_{1}$ and $x_{2}$ are the inductor current and the capacitor (output) voltage, respectively. $u$ is a binary variable, $u \in\{0,1\}$, which defines the switch position and plays the role of the control input. The inductor $L$, the capacitor $C$, and the source voltage $V_{\text {in }}$ are supposed to be known constants. The resistance $R$ is unknown, but is considered to be constant for analysis purposes.

A sliding surface $\sigma=0$ along which the system trajectory should slide and present desired dynamics is proposed as:

$$
\begin{aligned}
\sigma= & \int_{0}^{t}\left(G \int_{0}^{\tau}\left(V_{\text {in }}-u(s) x_{2}(s)\right) d s+G k_{p}\left(x_{2}(t)-V_{\text {ref }}\right)\right. \\
& \left.+G k_{i} \int_{0}^{\tau}\left(x_{2}(s)-V_{\text {ref }}\right) d s-g_{p} u(\tau)\right) d \tau=0
\end{aligned}
$$

with $u$ the switching policy as defined in (2). The constants $G, k_{p}$ and $k_{i}$ are design parameters of the controller that have to be adjusted to ensure the conditions for a sliding mode to exist. The scalar function $\sigma$ as in (11) has the form (9) with $k_{a}=1, k_{b}=0$ and,

$$
\begin{aligned}
\phi= & G \int_{0}^{\tau}\left(V_{\text {in }}-u(s) x_{2}(s)\right) d s+G k_{p}\left(x_{2}(t)-V_{\text {ref }}\right) \\
& +G k_{i} \int_{0}^{\tau}\left(x_{2}(s)-V_{\text {ref }}\right) d s .
\end{aligned}
$$


Hence, controller (2) and (11) can be implemented by means of a PWM making $d=\phi(\mathbf{x}) / g_{p}$. In some cases, the function $\phi$ may define a sliding surface $\phi=0$ by itself, however, this fact is not necessary for our purposes. This idea was first sketched heuristically in [17] for a normalised model of the boost converter which is equivalent to (10), and shown to be also useful for the boost inverter in [18]. The heuristic idea behind the fact of proposing $\phi$ as in (12) is the following one: when the system enters a sliding motion then $\sigma=0$, hence $\dot{\sigma}=0$, and $\phi-g_{p} u=0$. In the stationary state $u=\bar{u}$, with $\bar{u}$ constant. This was pursued by the controller proposed in [17]. In the present paper, all these facts, which were preliminarily sketched in [12], are analytically proven.

\subsection{Validity of the control methodology proposed}

Firstly, under certain design conditions, a sliding motion is shown to be present on the surface $\sigma=0$. Secondly, values for parameters $k_{i}$ and $k_{p}$ are chosen so that once the system trajectories slide on $\sigma=0$, after certain time, they will converge to the desired equilibrium, which basically means that $x_{2} \rightarrow V_{\text {ref }}$. Furthermore, a bound from below for the time the system trajectories need to achieve this control goal is obtained.

Proposition 1. Consider

$\sigma(\mathbf{x}, t)=\int_{0}^{t}\left[\phi(\mathbf{x}(\tau), \tau)-g_{p} u(\tau)\right] d \tau$.

Let $V_{\text {ref }}, V_{\mathrm{in}}, R, C, L, G, k_{p}, k_{i}, g_{p}$ be positive constants and $V_{\mathrm{ref}}>V_{\mathrm{in}}$. Let define

$t_{\sigma_{1}}=\frac{k_{p} V_{\mathrm{ref}}}{V_{\mathrm{in}}-k_{i} V_{\mathrm{ref}}}$.

If

$0<k_{i} \frac{V_{\text {ref }}}{V_{\text {in }}}<1$,

then any trajectory of system (10) with control

$u= \begin{cases}0 & \text { if } \sigma(\mathbf{x}, t)<0, \\ 1 & \text { if } \sigma(\mathbf{x}, t)>0,\end{cases}$

goes into a sliding motion on the surface $\sigma(\mathbf{x}, t)=0$ with $t>t_{\sigma_{1}}$.

Proof. From (13), the derivative of $\sigma$ along the trajectories of the system is:

$\dot{\sigma}(t)=\phi(\mathbf{x}(t), t)-g_{p} u(t)$.

The proof consists of two parts. First, the surface $\sigma=0$ will be shown to be locally attractive, that is, there is a subset $\mathcal{S}_{\sigma} \subset \mathbb{R}^{2}$ where $\sigma \dot{\sigma}<0[14,15]$. Second, any trajectory will be shown to hit the surface $\sigma=0$ within the set $\mathcal{S}_{\sigma}$.

For the first part of the proof, two cases are considered:

- Case 1: $\sigma<0$. According to the switching policy (16), $u=0$ and,

$$
\begin{aligned}
\dot{\sigma}(t)= & \left.\phi(\mathbf{x}(t), t)\right|_{u=0}=G \int_{0}^{t} V_{\text {in }} d t+G k_{p}\left(x_{2}(t)-V_{\text {ref }}\right) \\
& +G k_{i} \int_{0}^{t}\left(x_{2}(\tau)-V_{\text {ref }}\right) d \tau .
\end{aligned}
$$

Consequently, $\left.\dot{\sigma}\right|_{u=0}>0$ in the following set:

$$
\begin{aligned}
\mathcal{S}_{\sigma_{1}}:= & \left\{\mathbf{x} \in \mathbb{R}^{2}, t>0: k_{p}\left(x_{2}(t)-V_{\text {ref }}\right)\right. \\
& \left.+k_{i} \int_{0}^{t}\left(x_{2}(\tau)-V_{\text {ref }}\right) d \tau>-\int_{0}^{t} V_{\text {in }} d t\right\} .
\end{aligned}
$$

- Case 2: $\sigma>0$. In this case, $u=1$, and

$$
\begin{aligned}
\dot{\sigma}(t)= & \left.\phi(\mathbf{x}(t), t)\right|_{u=1}-g_{p} \\
= & G \int_{0}^{t}\left(V_{\text {in }}-x_{2}(\tau)\right) d \tau+G k_{p}\left(x_{2}(t)-V_{\text {ref }}\right) \\
& +G k_{i} \int_{0}^{t}\left(x_{2}(\tau)-V_{\text {ref }}\right) d \tau-g_{p} .
\end{aligned}
$$

Consequently, $\left.\dot{\sigma}\right|_{u=1}<0$ in the following set:

$$
\begin{aligned}
\mathcal{S}_{\sigma_{2}}:= & \left\{\mathbf{x} \in \mathbb{R}^{2}, t>0: k_{p}\left(x_{2}(t)-V_{\text {ref }}\right)+k_{i} \int_{0}^{t}\left(x_{2}(\tau)-V_{\text {ref }}\right) d \tau<g_{p}\right. \\
& \left.+\int_{0}^{t}\left(x_{2}(\tau)-V_{\text {in }}\right) d \tau\right\} .
\end{aligned}
$$

Thus, $\sigma \dot{\sigma}<0$ in the set $\mathcal{S}_{\sigma_{1}} \cap \mathcal{S}_{\sigma_{2}}:=\mathcal{S}_{\sigma}$, that is,

$$
\begin{aligned}
\mathcal{S}_{\sigma}= & \left\{\mathbf{x} \in \mathbb{R}^{2}, t>0:-\int_{0}^{t} V_{\text {in }} d t<k_{p}\left(x_{2}(t)-V_{\text {ref }}\right)\right. \\
& \left.+k_{i} \int_{0}^{t}\left(x_{2}(\tau)-V_{\text {ref }}\right) d \tau<g_{p}+\int_{0}^{t}\left(x_{2}(\tau)-V_{\text {in }}\right) d \tau\right\} .
\end{aligned}
$$

Therefore, on $\sigma=0$, a sliding motion takes place when the system trajectory hits the surface $\sigma=0$ within the set $\mathcal{S}_{\sigma}$.

Now, it is shown that any trajectory eventually hits the surface $\sigma=0$ at an $\mathbf{x}$ within the set $\mathcal{S}_{\sigma}$ and for $t>t_{\sigma_{1}}$.

Suppose that $\sigma<0$ at certain time, then $u=0$ and system (10) is described by

$\dot{x}_{1}=\frac{V_{\text {in }}}{L}, \dot{x}_{2}=-\frac{x_{2}}{R C}$,

then $x_{2} \rightarrow 0$. According to (17),

$\dot{\sigma}=\left.\phi\right|_{u=0} \rightarrow G t\left(V_{\text {in }}-k_{i} V_{\text {ref }}\right)-G k_{p} V_{\text {ref }}$.

Taking into account that condition (15) is satisfied, $\dot{\sigma}$ becomes positive for $t>t_{\sigma_{1}}$, with $t_{\sigma_{1}}$ as defined in (14). Therefore, after $t=t_{\sigma_{1}}$, $\sigma$ eventually increases until it gets zero, thus hitting the surface $\sigma=0$.

On the other hand, if $\sigma>0$ then system (10) is described by

$\dot{x}_{1}=\frac{V_{\text {in }}}{L}-\frac{x_{2}}{L}, \dot{x}_{2}=-\frac{x_{2}}{R C}+\frac{x_{1}}{C}$.

Then $\mathbf{x} \rightarrow \overline{\mathbf{x}}$, with $\overline{\mathbf{x}}=\left(\left(V_{\text {in }} / R\right), V_{\text {in }}\right)^{T}$. From (17), it is obtained that $\dot{\sigma}=\left.\phi\right|_{u=1}-g_{p}$.

Since $V_{\text {in }}<V_{\text {ref }}$ and $\mathbf{x} \rightarrow \overline{\mathbf{x}},\left.\phi\right|_{u=1}<0$ and consequently, $\dot{\sigma}$ will become strictly negative. Hence, at some time, $\sigma$ starts decreasing until it eventually crosses zero and hits $\sigma=0$. A bound from below for time at which $\sigma$ starts approaching zero can be obtained. Provided that condition (15) is satisfied and according to (20), it is concluded that $\overline{\mathbf{x}} \in \mathcal{S}_{\sigma}$ for $t>t_{\sigma_{2}}$ with,

$t_{\sigma_{2}}=\frac{k_{p}\left(V_{\text {ref }}-V_{\text {in }}\right)}{k_{i} V_{\text {in }}+\left(V_{\text {in }}-k_{i} V_{\text {ref }}\right)}$.

This implies that if $\sigma>0$, after $t>t_{\sigma_{2}}$, the system trajectory will hit $\sigma=0$.

Notice that $t_{\sigma_{1}}>t_{\sigma_{2}}$, provided that condition (15) is satisfied.

To conclude with, the trajectory reaches the surface $\sigma=0$ from any point $\mathbf{x} \in \mathbb{R}^{2}$ after $t=t_{\sigma_{1}}$ if condition (15) is satisfied. Besides, the system trajectory moves towards $\mathcal{S}_{\sigma}$ independently of the sign of $\sigma$, entering a sliding regime on $\sigma=0$. 
Remark 2. Proposition 1 does not assert that if the system trajectory goes into the sliding regime on the surface $\sigma=0$ then it remains there. The trajectory may leave the sliding mode. However, if this happens, the trajectory may eventually return to the surface.

In Proposition 1, the trajectories of system (10) are shown to reach $\sigma=0$ by means of control (16). Below, Proposition 3 states that once the trajectory hits $\sigma=0$, after certain time, it remains on it, due to the fact that it eventually converges to a asymptotically stable quasi-equilibrium on $\sigma=0$.

System dynamics (10) while evolving on $\sigma=0$ have the form $\dot{\mathbf{x}}=$ $f_{\sigma}(\mathbf{x})$, where $f_{\sigma}$ stands for the equivalent dynamics on $\sigma=0[14,15]$. Dynamics $f_{\sigma}(\mathbf{x})$ are obtained by substituting $u=u_{\mathrm{eq}}$ into system (10), with $u_{\mathrm{eq}_{\sigma}}$ the solution for $u$ of equation $\dot{\sigma}=\phi-g_{p} u=0$. Thus,

$u_{\mathrm{eq}_{\sigma}}(\mathbf{x})=\frac{\phi(\mathbf{x})}{g_{p}}$.

Consider the auxiliary state $x_{3}(t)=\int_{0}^{t}\left(x_{2}(\tau)-V_{\text {ref }}\right) d \tau$, consequently, the function $\phi$ can be rewritten as

$\phi(\mathbf{x})=G\left[L x_{1}+k_{p}\left(x_{2}-V_{\text {ref }}\right)+k_{i} x_{3}\right]$.

Hence, from (24) and (25) and (10), $\dot{\mathbf{x}}=f_{\sigma}(\mathbf{x})$ has the form:

$\dot{x}_{1}=\frac{V_{\text {in }}}{L}-\frac{G}{L g_{p}}\left[L x_{1} x_{2}+k_{i} x_{2} x_{3}+k_{p} x_{2}^{2}-k_{p} V_{\text {ref }} x_{2}\right]$,

$\dot{x}_{2}=-\frac{x_{2}}{R C}+\frac{G}{C g_{p}}\left[L x_{1}^{2}+k_{i} x_{1} x_{3}+k_{p} x_{1} x_{2}-k_{p} V_{\text {ref }} x_{1}\right]$,

$\dot{x}_{3}=x_{2}-V_{\text {ref }}$.

The quasi-equilibrium of system (10) on the switching surface $\sigma=0$, denoted by $\hat{\mathbf{x}}_{\sigma}$, such that $f_{\sigma}\left(\hat{\mathbf{x}}_{\sigma}\right)=0$ is obtained from (26) as

$\hat{x}_{\sigma, 1}=\frac{V_{\text {ref }}^{2}}{R V_{\text {in }}}, \quad \hat{x}_{\sigma, 2}=V_{\text {ref }}, \quad \hat{x}_{\sigma, 3}=\frac{g_{p} R V_{\text {in }}^{2}-G L V_{\text {ref }}^{3}}{R G k_{i} V_{\text {ref }} V_{\text {in }}}$.

The equilibrium $\hat{\mathbf{x}}_{\sigma}$ is a desired steady state for system (10).

Proposition 3. Let $V_{\mathrm{ref}}, V_{\mathrm{in}}, R, C, L, G, k_{p}, k_{i}, g_{p}$ be positive constants and $V_{\text {ref }}>V_{\text {in. If: }}$

$0<k_{i} \frac{V_{\text {ref }}}{V_{\text {in }}}<1$,

$$
\begin{aligned}
& \frac{1}{R^{2} V_{\text {ref }}^{2}}\left\{\left[g_{p} V_{\text {in }}+G V_{\text {ref }}\left(C R V_{\text {in }}-k_{p} V_{\text {ref }}\right)\right]\right. \\
& \left.\quad \times\left[g_{p} R V_{\text {in }}^{3}+G V_{\text {ref }}^{2}\left(k_{p} R V_{\text {in }}^{2}+L V_{\text {ref }}\left(2 V_{\text {in }}-k_{i} V_{\text {ref }}\right)\right)\right]\right\}-C G g_{p} k_{i} V_{\text {in }}^{3}>0,
\end{aligned}
$$

then the quasi-equilibrium point $\hat{\mathbf{x}}_{\sigma}(27)$ is locally asymptotically stable.

Proof. From (26), the characteristic polynomial of the Jacobian matrix of the equivalent dynamics on $\sigma=0$ evaluated at $\hat{\mathbf{x}}_{\sigma}$ is

$\lambda^{3}+a_{2} \lambda^{2}+a_{1} \lambda+a_{0}=0$,

with

$$
\begin{aligned}
& a_{0}=\frac{G k_{i} V_{\text {in }}}{g_{p} C L}, \\
& a_{1}=\frac{1}{g_{p} R C L V_{\text {in }} V_{\text {ref }}^{2}}\left\{g_{p} R V_{\text {in }}^{3}+G V_{\text {ref }}^{2}\left[k_{p} R V_{\text {in }}^{2}+L V_{\text {ref }}\left(2 V_{\text {in }}-k_{i} V_{\text {ref }}\right)\right]\right\}, \\
& a_{2}=\frac{1}{g_{p} R C V_{\text {in }}}\left[g_{p} V_{\text {in }}+G V_{\text {ref }}\left(R C V_{\text {in }}-k_{p} V_{\text {ref }}\right)\right] .
\end{aligned}
$$

Table 1

Parameters of the boost converter

\begin{tabular}{ll}
\hline$V_{\text {in }}$ & $10 \mathrm{~V}$ \\
$L$ & $2 \mathrm{mH}$ \\
$C$ & $33 \mu \mathrm{F}$ \\
$R$ & $25 \Omega$ \\
$V_{\text {ref }}$ & $20 \mathrm{~V}$ \\
\hline
\end{tabular}

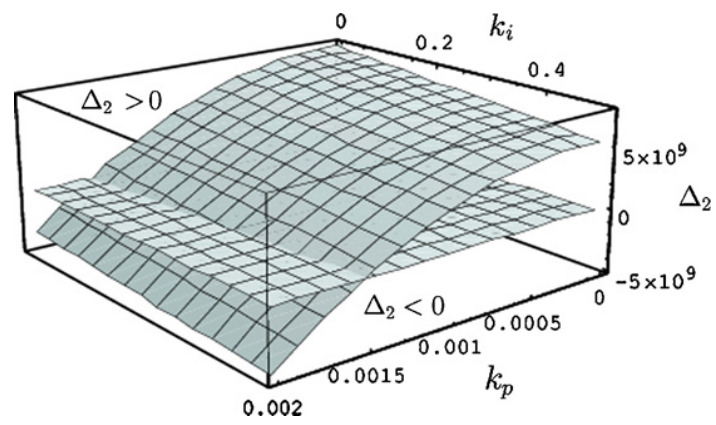

Fig. 4. Evaluation of the Routh-Hurwitz stability condition (28b) for the equilibrium $\hat{\mathbf{x}}_{\sigma}$ on $\sigma=0$.

The local asymptotic stability of $\hat{\mathbf{x}}_{\sigma}$ is assured by means of the Routh-Hurwitz criterion, that is, when

$a_{0}>0, a_{1}>0, \Delta_{2}=a_{1} a_{2}-a_{0}>0$.

The condition $a_{0}>0$ is directly satisfied. In addition, $a_{1}>0$ if condition (28a) is satisfied. $\Delta_{2}>0$ is precisely condition (28b).

To conclude with, once the trajectory hits $\sigma=0$, after certain time, it converges to the quasi-equilibrium $\hat{\mathbf{x}}_{\sigma}$ on $\sigma=0$.

Remark 4. The results of Propositions 1 and 3 throw light on the role of controller gains $k_{i}$ and $k_{p}$. On the one hand, $k_{i}$ assures the sliding motion on $\sigma=0$. On the other hand, the convergence time of the trajectories of system (10) towards $\sigma=0$ is regulated by means of the value of $k_{p}$ (through expression of time $t_{\sigma_{1}}$ ). Furthermore, the stability of the desired equilibrium on $\sigma=0$ is assured by means of $k_{i}$ and $k_{p}$.

\section{Simulations and experimental results}

In this section, the performance of the closed-loop system (10), (16), (11) is evaluated either in simulations or experimentally. In addition, it is experimentally shown that controller (16), (11) can indeed be implemented by using a PWM.

The parameters of the boost converter employed are shown in Table 1 . In addition, $g_{p}=0.05$ and $G=1$. Now, the controller parameters $k_{i}$ and $k_{p}$ must be chosen in order to satisfy conditions for the existence of a sliding motion on $\sigma=0$ and for the stability of the equilibrium point $\hat{\mathbf{x}}_{\sigma}$.

Firstly, a value for $k_{i}$ is chosen so that condition (15) is satisfied. From this condition, and for parameters shown in Table 1, $0<k_{i}<0.5$ is obtained. We will use $k_{i}=0.1$. By using this $k_{i}$, $k_{p}$ must be selected in order to satisfy condition (28b). For each value of $k_{i}$, a bound from above for $k_{p}$ can be obtained from condition (28b)(Fig. 4), that is, $\forall k_{i} \in(0,0.5)$, there exists one $\bar{k}_{p}$ such that $0<k_{p}<\bar{k}_{p}$, and condition (28b) is satisfied. For $k_{i}=0.1$, $\bar{k}_{p}=0.001505$. The value $k_{p}=0.001$ will be used.

The selected values of $k_{i}$ and $k_{p}$ assure the validity of the controller proposed as Propositions 1 and 3 establish. For the parameters used, $t_{\sigma_{1}}=2.5 \mathrm{~ms}$, with $t_{\sigma_{1}}$ the time the system trajectories need to reach $\sigma=0$. Time $t_{\sigma_{1}}$ obtained is low enough for the boost converter. 


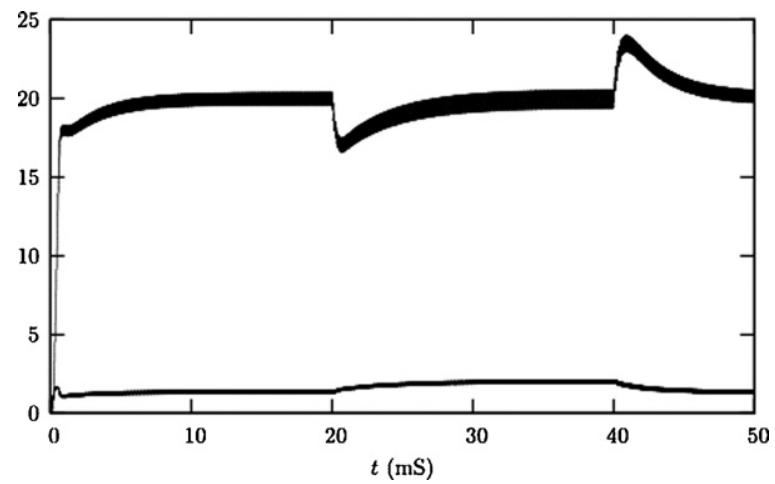

Fig. 5. Simulation of system (10) with the controller(16),(11) using a hysteresis band to limit the switching frequency. Top: output voltage. Bottom: inductor current.

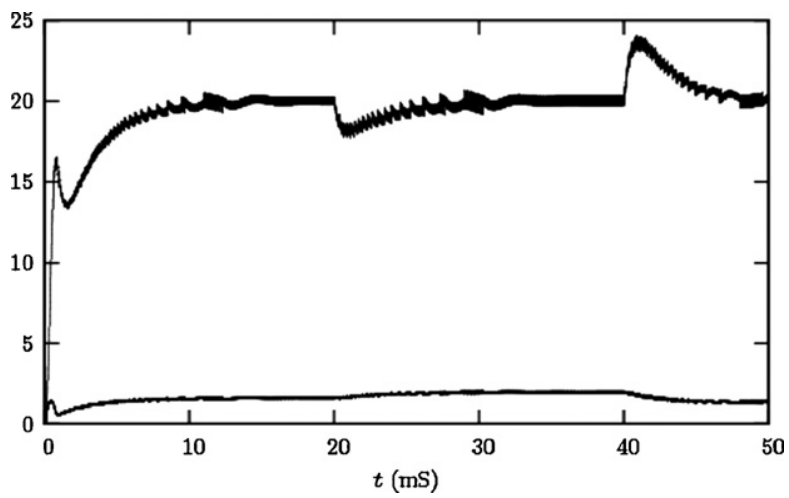

Fig. 6. Simulation of system (10) with the controller (16), (11) using a PWM and making $d=\phi / g_{p}$. Top: output voltage. Bottom: inductor current.

In simulations, the implementation of the sliding-mode-based controller is carried out by means of a PWM and by means of a hysteresis band. That is, system (10) is simulated by using the two control structures depicted in Fig. 1.

Fig. 5 shows the system performance when the controller (16), (11) is implemented using a hysteresis band to limit the switching frequency as in Fig. 1(a). In order to show the robustness of the controller, a sudden load change is introduced at $t=20 \mathrm{~ms}$ and at $t=40 \mathrm{~ms}$. It can be seen that the controller is highly robust under load variations.

Fig. 6 shows a simulation of the system with the same controller but this time, it was implemented following the scheme of Fig. 1(b), that is, using a PWM and making $d=\phi / g_{p}$. The switching frequency was set to $50 \mathrm{kHz}$. It can be seen that both alternatives of implementing the controller have a similar performance. However,

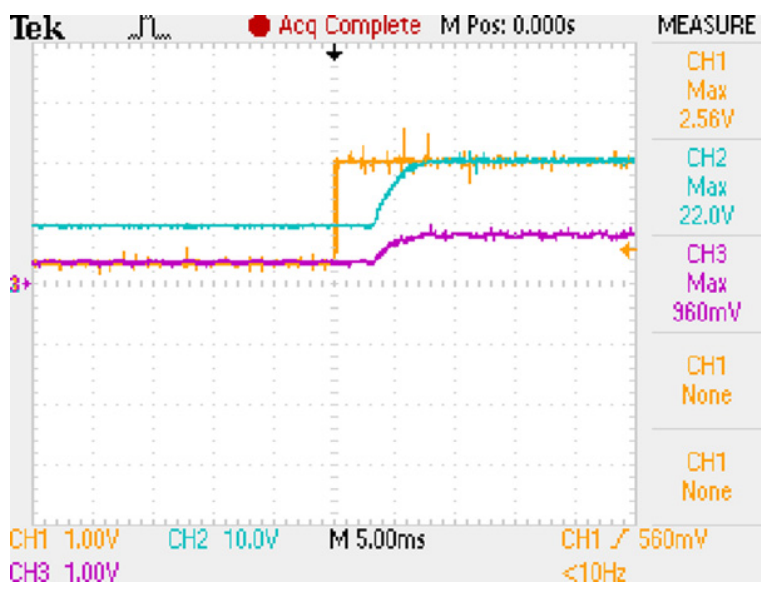

Fig. 8. Performance of the closed-loop system(10),(16),(11) under sudden reference change. Top: reference and output voltages. Bottom: inductor current.

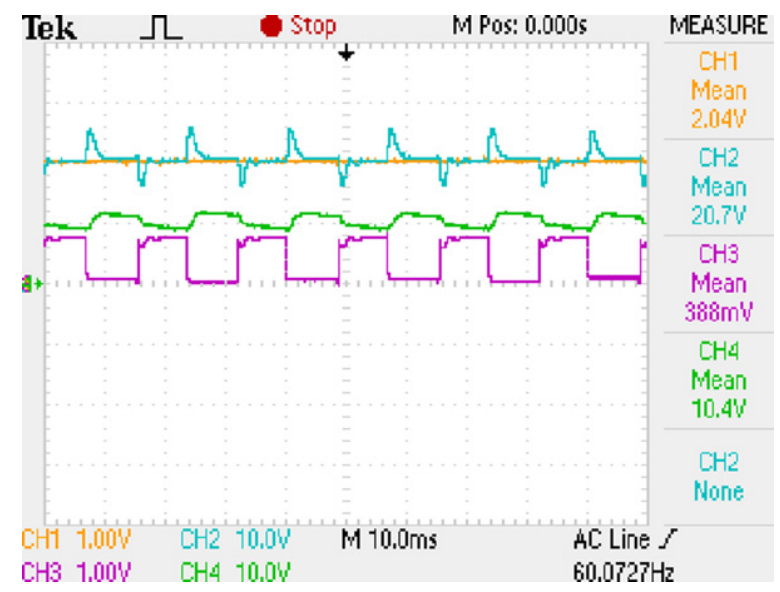

Fig. 9. Performance of the closed-loop system (10), (16), (11) under load variations. Top: reference and output voltages. Middle: inductor current. Bottom: load.

the PWM implementation is easier and has the great advantage of having a constant switching frequency. Therefore, for the real boostprototype, the implementation of the closed-loop system will be carried out by using a PWM.

In spite of its apparent complexity, the sliding-mode controller (16), (13), (12) can be implemented by means of the circuit depicted in Fig. 7. The circuit is rather simple because all signals operations involved in (12) can be carried out with operational amplifiers except the term $V_{\text {in }}-u x_{2}$, where a multiplier is apparently needed. However, it can be noticed from Fig. 3 that the term $V_{\text {in }}-u x_{2}$ actu-

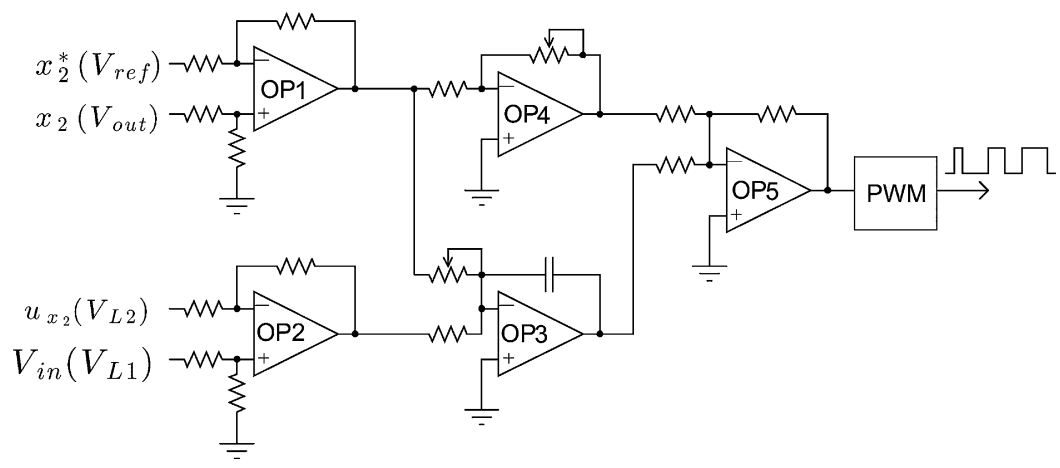

Fig. 7. Diagram of the circuit which implements controller (16), (13), (12). 
ally is the voltage across the inductor. This observation simplifies the circuit. As it is shown in the diagram, the controller only needs the output reference $V_{\text {ref }}$, the output voltage $x_{2}$ and the voltage across the inductor.

The controller has been experimentally evaluated using a PWM and the results are depicted in Figs. 8 and 9. In Fig. 8, the reference is suddenly changed from 0 to $20 \mathrm{~V}$. The output voltage follows this change reasonably fast. The system performance under periodic load variations between open circuit (no load) and $25 \Omega$ is shown in Fig. 9. Note that variations on the output voltage are very small and after the load changes it returns to its desired value. As it is expected, the inductor current does depend on the load.

These experimental results confirm the robustness of the controller, which was previously observed in simulations.

\section{Conclusions}

A class of easy-to-implement sliding-mode controllers for power converters has been proposed. The design process has been based on the relation between average control and sliding-mode control. A new kind of sliding surfaces which can be directly implemented by means of a pulse-width modulator has been proposed. The sliding-surface-type proposed is built based on other surface in which the system has desired dynamics. The obtained controller exhibits a constant switching frequency which can be selected by the designer. To show the feasibility of the control methodology proposed, it has been applied to the boost converter either in simulatios or experimentally in a real prototype. This example shows that the controller-type proposed has the usual high performance and robustness under large parameters variations of sliding-mode controllers. In addition, it has a constant switching frequency and is easy to implement keeping the overall circuit reliability.

\section{Acknowledgment}

This work was partially supported by CONACYT Mexico under contract 44969.

\section{References}

[1] V.I. Utkin, J. Guldner, J. Shi, Sliding Mode Control in Electromechanical Systems, 1 st ed., CRC, 1999.
[2] P. Mattavelli, L. Rossetto, G. Spiazzi, P. Tenti, A general-purpose sliding-mode controller for dc/dc converter applications, in: Proceedings of the 24th Power Electronics Specialists Conference, Seattle, USA, 1993, pp. 609-615.

[3] V.M. Nguyen, C.Q. Lee, Tracking control of buck converter using sliding mode with adaptive hysteresis, in: Proceedings of the 26th Power Electronics Specialists Conference, Atlanta, USA, 1995, pp. 1086-1093.

[4] V.M. Nguyen, C.Q. Lee, Indirect implementation of sliding mode control law in buck type converters, in: Proceedings of the IEEE Applied Power Electronics Conference, 1996, pp. 111-115.

[5] R. Ramos, D. Biel, E. Fossas, F. Guinjoan, A fixed-frequency quasi-sliding control algorithms: application to power inverters design by means of FPGA implementation, IEEE Trans. Power Electron. 18 (1) (2003) 344-355.

[6] S.C. Tan, Y.M. Lai, K.H. Cheung, C.K. Tse, On the practical design of a sliding mode voltage controlled buck converter, IEEE Trans. Power Electron. 20 (2) (2005) 425-437.

[7] S.C. Tan, Y.M. Lai, C.K. Tse, K.H. Cheung, A fixed-frequency pulsewidth modulation based quasi-sliding-mode controller for buck converters, IEEE Trans. Power Electron. 20 (6) (2005) 1379-1392.

[8] S.C. Tan, Y.M. Lai, C.K. Tse, Adaptive feedforward and feedback control schemes for sliding mode controlled power converters, IEEE Trans. Power Electron. 21 (1) (2006) 182-192.

[9] S.C. Tan, Y.M. Lai, C.K. Tse, Implementation of pulse-width-modulation based sliding mode controller for boost converters, IEEE Power Electron. Lett. 3 (4) (2006) 130-135.

[10] S.C. Tan, Y.M. Lai, C.K. Tse, A unified approach to the design of PWM-based sliding-mode voltage controllers for basic DC-DC converters in continuous conduction mode, IEEE Trans. Circuits Syst. I: Regular Papers 53 (8) (2006) 1816-1827.

[11] R. Venkataramanan, A. Sabanovic, S. Cuk, Sliding mode control of dc-to-dc converters, in: Proceedings of the IEEE Industrial Electronics Conference (IECON), San Francisco, USA, 1985, pp. 251-258.

[12] D. Cortés, Ja. Álvarez, E.M. Navarro-López, C. Castro, A class of easy-toimplement sliding-mode controllers with constant switching frequency for power converters, in: Proceedings of the IEEE International Symposium on Industrial Electronics, Vigo, Spain, 2007, pp. 731-735.

[13] D. Cortés, Generación de Voltajes de Corriente Alterna mediante Convertidores de Alta Frecuencia de Conmutación, PhD Thesis, Centro de Investigación Científica y de Educación Superior de Ensenada, B.C., Mexico, November 2004.

[14] A.F. Filippov, Differential Equations with Discontinuous Right-hand Sides, Kluwer Academic Publishers, Dordrecht, 1988.

[15] V.I. Utkin, Sliding Modes in Control and Optimization, 1st ed., Communications and Control Engineering Series, Springer Verlag, New York, 1991.

[16] H. Sira-Ramírez, A geometric approach to pulse width modulated contro in nonlinear dynamical systems, IEEE Trans. Automat. Control 34 (3) (1989) 184-187.

[17] D. Cortés, Ja. Álvarez, Jq. Álvarez, Robust control of the boost converter, in: Proceedings of the IEEE Industrial Electronics and Control Applications (ICIECA) Quito, Ecuador, 2005.

[18] D. Cortés, N. Vázquez, Ja. Álvarez, Robust control of the boost converter, in: Proceedings of the IEEE Industrial Electronics Conference (IECON), Paris, France, 2006, pp. 1890-1895. 